\title{
Fuzzy identification of bioactive components for different efficacies of a medicinal herb by BP neural network association analysis of UPLC-Q-TOF/MSE and integrated effects
}

Jia-Qian Chen

Shaanxi University of Chinese Medicine

Yan-Yan Chen

Shaanxi University of Chinese Medicine

Xia Du

Shaanxi Academy of Traditional Chinese Medicine

Hui-Juan Tao

Nanjing University of Chinese Medicine

Zong-Jin Pu

Nanjing University of Chinese Medicine

Xu-Qin Shi

Nanjing University of Chinese Medicine

Shi-Jun Yue

Shaanxi University of Chinese Medicine

Gui-Sheng Zhou

Nanjing University of Chinese Medicine

Er-Xin Shang

Nanjing University of Chinese Medicine

Yu-Ping Tang ( $\nabla$ yupingtang@sntcm.edu.cn )

Shaanxi University of Chinese Medicine

Jin-Ao Duan

Nanjing University of Chinese Medicine

\section{Research Article}

Keywords: Herb, Efficacy, Bioactive component, Fuzzy identification, Integrated effect, Rhubarb

Posted Date: February 15th, 2022

DOI: https://doi.org/10.21203/rs.3.rs-1319361/v1 
License: (c) (i) This work is licensed under a Creative Commons Attribution 4.0 International License. Read Full License 


\section{Abstract}

Background: The multicomponent nature of a medicinal herb leads to multiple efficacies via numerous metabolic byproducts, potential molecular interactions and targets, which makes a network-oriented approach preferable in the preliminary stage of herbal medicine research. In this study, we aim to develop a generally applicable strategy to fuzzily identify bioactive components for different efficacies by back propagation artificial neural network (BP-ANN) association analysis of ultra-performance liquid chromatography/quadrupole time-of-flight mass spectrometry for every data (UPLC-Q-TOF/MS ${ }^{\mathrm{E}}$ ) and integrated effects.

Methods: Through applying fuzzy chemical identification, most components in an herb can be classified into different chemical groups. Meanwhile the integration effect values of herbal different efficacies may be obtained by animal experiment evaluation and multi-attribute comprehensive indexes. Then BP-ANN is used for association analysis of herbal components and different efficacies by correlating the component contents determined from UPLC-Q-TOF/MS ${ }^{\mathrm{E}}$ profiling and the integration effect values. So, the effect contribution of one type of components might be totaled to demonstrate herbal universality and individuality characters for different efficacies.

Results: In the case of rhubarb, it suggested that combined anthraquinones, flavanol and its polymers might be the universality character to the multi-functional properties while stilbene glycosides, anthranone and its dimers, free anthraquinones, chromones, gallic acid and gallotannins, butyrylbenzene and its glycosides contributed to the individuality characteristics.

Conclusion: This novel methodology was successfully applied to investigate and differentiate the bioactive components of rhubarb. These efforts will promote our recognition and understanding of the bioactive components in rhubarb and provide scientific evidence to support the expansion of its use in clinical applications and the further development of some products based on this medicinal herb. And this approach should also be useful for investigating the bioactive constituents of other medicinal herbs or natural functional products.

\section{Background}

As one of the oldest continuously practiced systems of traditional medicine in the world, herbal medicines have an application history of several thousand years and their worldwide utilization has increased recently in developing and developed countries [1, 2]. In China, nearly half of all commercial drugs are botanicals that have been developed from herbal medicines and widely applied for the treatment of various chronic diseases including cardiovascular disease and cancer [3]. Isolation and purification of active ingredients from natural products especially from herbal medicines is also regarded as a successful approach to novel drug identification and development. However, the pharmacologically active ingredients of herbal medicines as phytocomplex are not always the original natural molecules, but may be their host-specific metabolites or molecular complexes formed from some different types of 
natural compounds ${ }^{2}$. This complexity brings significant scientific challenges in the study of herbal active ingredients. The multicomponent nature of herbal medicines generates multiple potential molecular interactions, multiple targets and numerous metabolic byproducts, and further leads to multiple efficacies, which suggests that a conventional reductionist approach will have limitations in identifying bioactive components, making a more holistic, network-oriented approach preferable.

Although many methods have been recently developed to investigate the bioactive components for main efficacy or pharmacological function of herbal medicine [4-11], nobody tries to touch upon that of multiple efficacies because each of herbal efficacies corresponds many different bioactive components. In this study, we aimed to develop a generally applicable approach and methodology to fuzzily identify bioactive components for different efficacies of a medicinal herb by back propagation artificial neural network (BP-ANN) association analysis of ultra-performance liquid chromatography/quadrupole time-offlight mass spectrometry for every data (UPLC-Q-TOF/MS ${ }^{\mathrm{E}}$ ) and integrated effects (Figure 1). Such a strategy was predominantly inspired by an idea that most components in an herb can usually be classified into one of various chemical structure types, and they may be attributed to different chemical groups by a fuzzy chemical identification strategy without needing identification of their exact chemical structures, and further their relative contents in herbal different extracts could be determined by UPLC-Q$\mathrm{TOF} / \mathrm{MS}^{\mathrm{E}}$. Meanwhile the integration effect values of herbal different efficacies might be obtained by animal experiment evaluation and multi-attribute comprehensive index analysis. Subsequently, BP-ANN was used for association analysis of herbal components and different efficacies by correlating the component contents and integration effects. So, contributions of each component on herbal different efficacies were quantitatively characterized, and the effect contribution of one type of components might also be totaled to demonstrate its universality and individuality characters for herbal different efficacies.

Following our previous attempt $[3,12-15]$, this novel approach was successfully applied to investigate and differentiate the bioactive components of rhubarb (also named Rhei or Dahuang). Rhubarb, one of the most ancient and important medicinal herbs in many countries, belongs to the Rheum L. genus from the Polygonaceae family, and its application can be traced back to 270 BC in Chinese "Shen Nong Ben Cao Jing". Rhubarb is one of the most effective laxatives and is widely used in the treatment of intestinal constipation throughout the world. It is not only officially listed in the Chinese Pharmacopoeia but also appears in the British Pharmacopoeia and European Pharmacopoeia [16]. Rhubarb was commonly used for "removing accumulation with purgation" (E1), "clearing heat and purging fire" (E2), "cooling blood and detoxifying" (E3), "eliminating blood stasis to remove obstruction in channels" (E4) and "disinhibiting dampness and removing jaundice" (E5) recorded as main efficacies in many Chinese medicine classics including Chinese Pharmacopoeia. And it also exhibited multiple pharmacological effects in modern research such as laxative [17], choleretic [18], renal and hepatic protective [19, 20], antineoplastic [21], hypolipidemic [22] as well as promoting blood circulation and removing blood stasis [23]. Although many reports $[24,25]$ have attributed the biological activity of rhubarb to several classes of compounds, such as anthraquinones, flavonoid and its glycosides, stilbene glycosides, chromones, gallic acid and gallotannins, butyrylbenzene and its glycosides, naphthalene glycosides, and especially free and 
combined anthraquinones are regarded as the primary bioactive components of rhubarb, the contributions of these components to different efficacies of the herb are still unknown. Therefore, the approach and methodology developed in this study were used to comprehensively investigate the bioactive components in rhubarb and to quantitatively differentiate them with respect to their contribution to each of five main efficacies. These efforts will promote our recognition and understanding of the bioactive components in rhubarb and provide scientific evidence to support the expansion of its use in clinical applications and the further development of some products based on this medicinal herb.

\section{Materials And Methods}

\section{Materials and Reagents}

Anhydrous ethanol (analytical grade $\geq 99.7 \%$ ), methanol (HPLC grade) and dimethyl sulfoxide (DMSO, $\geq$ 99.9\%) were purchased from Merck. Lipopolysaccharide (LPS, L2880) was from Sigma. a-Naphthyl isothiocyanate (ANIT, E1909091) was from Aladdin. White sugar (20180404), Yili whole milk powder (20180409), Jinluo edible lard (20180317), Yujiangyuan virgin olive oil (20191008) and adrenalin hydrochloride (Adr, $1 \mathrm{mg} / \mathrm{mL}$ for livestock, 20190307) were bought from the market. Drugs included Cisapride Tablet (20180518, Anglikang), Compound Danshen Dripping Pill (170912, Tasly), Ursodeoxycholic Acid Capsule (L19001A, Kangzhe) and 28 chemical references of rhubarb (purity $\geq 98 \%$, Nanjing Jin Yibai Biological Technology Co., Ltd., details listed in Table S2). Antibodies used for western blotting were the same as our preceding research [26]. Mouse enzyme-linked immunosorbent assay (ELISA) kits covering MTL, SS, VIP, AchE, TG, Na+ ${ }^{+} \mathrm{K}^{+}-$ATPase, TNF-a, IL-1 $\beta$, IL-6, HSP-70, (T-)SOD, NO, $\mathrm{TXB}_{2}$, 6-keto-PGF ${ }_{1 a}, \mathrm{PGE}_{2}, \mathrm{ET}-1, \mathrm{Mg}^{2+}, \mathrm{Ca}^{2+}, \mathrm{MDA}, \mathrm{GSH}, \mathrm{ALT}, \mathrm{AST}, \mathrm{ALP}, \mathrm{GST}, \mathrm{GGT}$, TBIL, DBIL and TBA were all obtained from Shanghai Enzyme-linked Biotechnology Co., Ltd. Kits of coagulation four indices of TT, PT, APTT and FIB were from Steellex. Serum and tissue Fe ${ }^{3+}$ test kits (colorimetry, A039-1-1, A039-2-1) were from Nanjing Jiancheng Bioengineering Institute.

\section{Main Equipment}

The laboratory is equipped with micro-plate reader (EnSpire, Molecular Devices), protein electrophoresis apparatus (PowerPac, BioRad), gel imager (\#1708195, BioRad), platelet aggregation/coagulation analyzer (LG-PABER-1, Steellex), UHPLC Acquity ${ }^{\mathrm{TM}}$ system coupled to Synapt ${ }^{\mathrm{TM}} \mathrm{Q}-\mathrm{TOF}$ mass spectrometer (Waters Corp.), etc.

\section{Preparation of Rhubarb Liquids}

The rhubarb was identified by Professor Hui Yan (Department of Pharmacognosy, Nanjing University of Chinese Medicine) as Rheum tanguticum Maxim. ex Balf. (No. NJUTCM-20171015) from Gannan Prefecture, Gansu Province, China. According to the data mining of multifunctional preparation and usage of rhubarb before [27], it was found that the most commonly used extraction methods were water and wine decoction repeated twice for 15-200 $\mathrm{min}$. So, we designed the rhubarb sample library from two aspects of 9 different concentrations of ethanol-water (EW) solvents and 2 extraction times. Short time 
(S) was 10 min for the first decoction and 5 min for the second while long time (L) was 120 min for the first and $80 \mathrm{~min}$ for the second. A total of 18 rhubarb groups were set up including water-S, water-L, 10\% EW-S, 10\% EW-L, 20\% EW-S, 20\% EW-L, 35\% EW-S, 35\% EW-L, 50\% EW-S, 50\% EW-L, 65\% EW-S, 65\% EW-L, $80 \%$ EW-S, $80 \%$ EW-L, 90\% EW-S, 90\% EW-L, ethanol-S, ethanol-L. The highest dose of Chinese Pharmacopoeia was selected to be converted to $1.95 \mathrm{~g} / \mathrm{kg}$ for mouse dosage. The process was to precisely weigh rhubarb powder passing through No.4 Pharmacopoeia sieve, fully soak with 2 times the amount of extraction solvent and then add 8 times the amount of corresponding solvent to boil out by reflux twice. After merging two filtrates while they were hot, the extracting solution was concentrated till no alcohol taste using rotatory evaporation $\left(50^{\circ} \mathrm{C}\right)$, and finally diluted with water as the suspension of $0.195 \mathrm{~g} / \mathrm{mL}$ for use.

\section{Pretreatment of Rhubarb Samples and Chemical References}

Rhubarb samples: Before vacuum concentration, $1 \mathrm{~mL}$ of each liquid was pipetted and accurately added into the homologous solvent to a concentration of $0.03 \mathrm{~g} / \mathrm{mL}$. Then $100 \mu \mathrm{L}$ of each sample was mixed as the quality control (QC) sample. Reference solution: The 28 chemical references were critically weighed 1 $\mathrm{mg}$ each adding with a trace amount of DMSO for hydrotropy and methanol till $1 \mathrm{~mL}$, followed by ultrasound to become the transparent solution. $100 \mu \mathrm{L}$ of each mother liquor was added to $1 \mathrm{~mL}$ of methanol for dilution, and then $100 \mu \mathrm{L}$ of each dilution was taken as the mixing standard sample. All above samples were placed in HPLC glass vials through microfiltration membrane $(0.22 \mu \mathrm{m})$ and stored at $4^{\circ} \mathrm{C}$ for testing.

\section{UPLC-Q-TOF/MSE Profiling}

In order to make the relative content determination of liquid ingredients more accurate, the acquisition time of mass spectrometry scanning was changed from $0.3 \mathrm{~s}$ to $0.1 \mathrm{~s}$, and the wavelength range of ultraviolet (UV) detection was set at $190-420 \mathrm{~nm}$. The phase A was water containing $0.1 \%$ formic acid and $B$ was acetonitrile. We optimized the gradient elution program: 0 2 min, 3 10\% B; 2 5 min, 10 15\% B; 5 8 min, 15\% B; 8 11 min, 15 23\% B; 11 16 min, 23 30\% B; $16 \sim 20 \mathrm{~min}, 30 \sim 60 \%$ B; 20 24 min, $60 \sim 80 \% \mathrm{~B} ; 24 \sim 25 \mathrm{~min}, 80 \sim 3 \%$ B. Remaining device parameters were the same as we described previously [28]. At the beginning of the analytical batch, QC sample was continuously injected 5 times to balance the system. Then 18 rhubarb samples were injected successively, and each sample was parallel for 3 times. Regular injection of corresponding blank solvent and QC samples could be used to evaluate the reliability and repeatability of instrument status throughout the workflow sequence. Under the same conditions, 28 rhubarb reference solution and mixing standard samples were detected in turn.

\section{Fuzzy Chemical Identification}

By the fuzzy chemical identification method [13], TCM components can be identified quickly, in which the core is to classify uncertain ones because compounds with the same mother nuclei possess similar action properties and interaction rules. It not only avoids some defects such as complex identification procedures of a great many components, scarcity of chemical references, but also reflects the vague and 
holistic view of traditional Chinese medicine (TCM). It needs to be implemented in conjunction with the full-spectrum information database of rhubarb (Table S1) we established through querying a large number of relevant literature reports and network platforms such as TCMSP (tcmspw.com), SymMap (www.symmap.org) and PubChem (pubchem.ncbi.nlm.nih.gov) for nearly 300 chemical compounds.

Under the premise of selecting different types of known components with high contents in rhubarb as the reference, we summarized the peak order, fragmentation information and pathways of different categories, and then established corresponding compound group networks to recognize unknown components rather than to confirm all components necessarily.

\section{Mouse Models and Determination of Pharmacodynamic Indexes}

BALB/c mice, weighing $22 \pm 2 \mathrm{~g}$, were purchased from Shanghai Sippr-BK Laboratory Animal Co., Ltd. (license number: SCXK (Hu) 2018-0006). Adaptive feeding environment and all animal related procedures were strictly in accordance with the criteria of the Animal Ethics Committee of Nanjing University of Chinese Medicine. These mice were free to eat conventional food and drink water. Before modeling, they were stratified on the basis of their weight and labeled, and were randomly divided into 21 groups including the control, model, positive and 18 rhubarb-treated groups.

\section{Constipation Model for Evaluation of E1 and E2}

The constipation model with gastrointestinal accumulated heat induced by dyspepsia was based on our established method [26]. The positive drug was Cisapride Tablet for the treatment of gastrointestinal dynamic diseases, and the maximum dosage was $3.9 \mathrm{mg} / \mathrm{kg}$ converted for mice. We divided 168 male mice (No. 20180006004662) into groups of 8 on average. From the first day of modeling, besides the control group, $0.8 \mathrm{~mL}$ of self-made high-calorie feed mixed by sugar, milk powder and lard [26] was additionally given to each mouse by intragastric administration 3 times a day at an interval of $6 \mathrm{~h}$, modeling for 7 consecutive days but changing into twice a day on the 6th and 7th days. Positive and rhubarb-treated groups were administered with corresponding drug liquids $(0.01 \mathrm{~mL} / \mathrm{g})$ in the morning of the 6th to 8th day while the control and model groups were intragastrically given isodose water at the same time. After $30 \mathrm{~min}$ of administration on day 8, all mice were intragastrically given Indian ink (0.01 $\mathrm{mL} / \mathrm{g}$ ) and immediately placed in the metabolic cages alone to observe defecation characteristics of first black stool time, the number of black stools and fecal weights within $12 \mathrm{~h}$. On the 9 th day, we weighed these mice, collected their serum, and then dissected $0.5 \mathrm{~cm}$ of duodena behind the pylorus, stomachs, colons and colonic contents. Biochemical index levels of MTL, SS, VIP, TG in mouse serum and AchE, $\mathrm{Na}^{+}-\mathrm{K}^{+}-\mathrm{ATPase}, \mathrm{TNF}-\mathrm{a}, \mathrm{IL}-1 \beta$ in duodenal tissues were detected by ELISA, and expressions of common inflammatory proteins in the colon covering p-NF-KB p65, NF-KB p65, p-p38, p38, p-ERK, ERK, p-JNK, JNK and TLR4 were determined by western blotting as depicted previously [26].

\section{Blood Stasis Syndrome for Evaluation of E3 and E4}


The blood stasis syndrome induced by noxious heat could be established by LPS-induced inflammation combined with Adr-induced Qi stagnation [29]. Existing literature reports basically used LPS to simulate the process of exogenous thermal toxicity, and its modeling dose for mice was $3 \mathrm{mg} / \mathrm{kg}$ prepared by $0.9 \%$ normal saline (NS). Adr injection for livestock would assist to achieve the effect of blood stasis in a short time, and its combined dosage was determined as $5 \mathrm{mg} / \mathrm{kg}$ through our continuous exploration to greatly reduce the mortality of mice. In this model, Compound Danshen Dripping Pill was chosen as the positive drug (105.3 mg/kg for the maximum dose of mice), which was often used clinically to regulate Qi and activate blood circulation. We separated 252 female mice (No. 20180006007816) into 12 mice in each group. On the first day of modeling, except injecting isodose $0.9 \%$ NS for the control group, all mice were intraperitoneally injected with LPS at $0.01 \mathrm{~mL} / \mathrm{g}$ for the first time and their anal temperatures should be measured punctually $4 \mathrm{~h}$ later. If the temperatures were abnormal, the model was successful, and then corresponding drug liquids were administered once every night $(0.01 \mathrm{~mL} / \mathrm{g}$, isodose water given to the control and model groups) for 6 consecutive days. On the 5th day, mice were subjected to LPS at 0.01 $\mathrm{mL} / \mathrm{g}$ by intraperitoneal injection and tested for anal temperatures $4 \mathrm{~h}$ later again. On the 6th day, they were given two subcutaneous injections of Adr at $0.005 \mathrm{~mL} / \mathrm{g}$ with a four-hour interval while the control group was accordingly injected with $0.9 \%$ NS. After fasting for at least $12 \mathrm{~h}$, they were weighed and sacrificed on day 7 . We randomly collected orbital blood of six mice in each group first, added $3.2 \%$ sodium citrate anticoagulant into their blood (1:9, v/v) immediately, and pipetted plasma by centrifugation to detect coagulation four indices of TT, PT, APTT and FIB. Secondly, serum from the remaining mice in each group was collected for ELISA levels of HSP-70, SOD, NO, TNF- $a$, IL-1 $\beta$, IL-6, TXB ${ }_{2}$, 6-keto-PGF ${ }_{1 a}, \mathrm{PGE}_{2}, \mathrm{ET}-1, \mathrm{Mg}^{2+}$ and $\mathrm{Ca}^{2+}$. Thirdly, all mice needed precise weighing of their spleens and thymuses. In addition, colon tissues were used to detect expression levels of the above 9 common inflammatory proteins by western blotting likewise.

\section{Cholestasis for Evaluation of E5}

ANIT can cause acute liver injury and has been used to establish the model of cholestatic jaundice in many studies. Through pretesting, we determined its modeling dose of $75 \mathrm{mg} / \mathrm{kg}$ for mice. The positive drug was Ursodeoxycholic Acid Capsule in this experiment, which could be cholagogic effectively at the maximum mouse equivalent dosage of $91 \mathrm{mg} / \mathrm{kg}$. Male mice (No. 20180006009949) were separated into an average of 8 per group. Positive and rhubarb groups were treated by prophylactic administration $(0.01$ $\mathrm{mL} / \mathrm{g}$ ) once a day for 3 days [18] while the control and model groups were given the same dose of water. On the 4th day, all mice suffered intragastrically $0.01 \mathrm{~mL} / \mathrm{g}$ of ANIT that dissolved in olive oil except the control group (equal dose of olive oil), and were administered with corresponding drug liquids once after $6 \mathrm{~h}$. They were given drug liquids again on the fifth night and fasted overnight. On day 6 (i.e., $48 \mathrm{~h}$ after ANIT modeling), we weighed them, drawn blood to store serum, and then took the whole livers and gallbladders for exact weighing respectively. Test kits were applied to detect levels of T-SOD, MDA, GSH, $\mathrm{Fe}^{3+}$ in mouse liver and ALT, AST, ALP, GST, GGT, TBIL, DBIL, TBA, $\mathrm{Fe}^{3+}$ in serum. The liver homogenate was prepared as follows: Each of liver tissues of about $50 \mathrm{mg}$ was added with $0.9 \% \mathrm{NS}(20 \mu \mathrm{L} / \mathrm{mg})$ and two steel balls, and put into an automatic grinding machine for $3 \mathrm{~min}$. The homogenate was placed on the ice for $30 \mathrm{~min}$ and centrifuged at $4^{\circ} \mathrm{C}$ to get the supernatant for determination. 


\section{BP neural network correlation method}

The relative content of each component in 18 rhubarb samples was correlated with 5 integration effects severally using BP neural network. It mainly consisted of three steps. (1) Data normalization: $y=(x-$ MinValue)/ (MaxValue - MinValue), where $x$ and $y$ were the values before and after conversion, and MaxValue and MinValue were the maximum and minimum values in samples. (2) Data set division: taking all component contents as input and corresponding integration effect values as output, and all of 1 18 group data as training samples, among which $90 \%$ EW-L, ethanol-S and ethanol-L groups were taken as test samples. (3) Quantity-effect correlation: based on BP neural network algorithm, adjusting various parameters to optimize the model for relevant investigation between components and efficacies, so as to reveal the influence of each component on the overall effect.

\section{Statistical analysis}

Experimental data were processed by GraphPad Prism 7.0 and SPSS 22.0, and expressed as "mean \pm standard deviation (SD)". One-way analysis of variance or independent sample $t$-test was carried out to compare the data between groups. Bilateral $P$ value less than 0.05 was considered to be statistically significant.

\section{Results And Discussion}

\section{Semi-Quantitative and Qualitative Analysis of Rhubarb Components Based on UPLC-Q-TOF/MSE and Fuzzy Chemical Identification}

The mixing standard sample basically achieved good separation within 25 min, which could provide a reference for qualitative analysis of various compounds. In PCA plots, QC samples were all close to the center of the Hotelling T2 ellipse, while 18 rhubarb groups were obviously separated but the same group of repeated sampling was clustered together. These indicated the stability of the UPLC-Q-TOF/MSE method (Figure S1). Combined with retention time (RT) and total ion chromatograms of three batches of rhubarb liquids, we screened out 108 unknown components with high responsiveness and different variation trends from 18 rhubarb samples. Peak areas, that is, relative contents of all components, were listed in Tables S3-5 calculated by EZinfo 2.0 software.

Furthermore, according to $\mathrm{MS}^{\mathrm{E}}$ characteristic fragmentation patterns of references, 25 components were confirmed via matching the 108 components. With reference to the full-spectrum information database of chemical compounds of rhubarb in Table S1, we constructed compound group networks of different categories (Figure 2) to classify the remaining components (Table 1). 
Table 1

Classification of 108 rhubarb components on the basis of fuzzy chemical identification.

\begin{tabular}{|c|c|c|}
\hline Category凶 & $\begin{array}{l}\text { Confirmed } \\
\text { component (No., } \\
\text { RT) }\end{array}$ & Remaining component attribution (RT) \\
\hline \multirow[t]{5}{*}{ Rh-01 } & $\begin{array}{l}\text { Aloe-emodin (90, } \\
17.70)\end{array}$ & \multirow[t]{5}{*}{68 (12.58), 78 (14.51), 93 (18.13), 96 (18.46), 99 (19.27) } \\
\hline & Rhein $(95,18.33)$ & \\
\hline & $\begin{array}{l}\text { Emodin (100, } \\
\text { 19.99) }\end{array}$ & \\
\hline & $\begin{array}{l}\text { Chrysophanol (102, } \\
21.24)\end{array}$ & \\
\hline & $\begin{array}{l}\text { Physcion (105, } \\
22.00)\end{array}$ & \\
\hline \multirow[t]{7}{*}{ Rh-02 } & $\begin{array}{l}\text { Aloe-emodin } 8-0-\beta \\
\text { D-glucoside }(30 \\
6.31)\end{array}$ & \multirow[t]{7}{*}{$\begin{array}{l}10(3.11), 22(4.76), 28(5.65), 36(6.97), 49(9.84), 52(10.28), 55 \\
(10.64), 64(11.67), 80(15.11), 83(15.72), 86(16.24), 89(17.48), \\
92(17.76), 97(18.94)\end{array}$} \\
\hline & $\begin{array}{l}\text { Rhein 8-O- } \beta \text {-D- } \\
\text { glucoside }(32,6.60)\end{array}$ & \\
\hline & $\begin{array}{l}\text { Emodin } 1-O-\beta-D- \\
\text { glucoside }(58 \\
\text { 10.97) }\end{array}$ & \\
\hline & $\begin{array}{l}\text { Chrysophanol 1-O- } \\
\beta \text {-D-glucoside (72, } \\
13.50)\end{array}$ & \\
\hline & $\begin{array}{l}\text { Emodin } 8-O-\beta \text {-D- } \\
\text { glucoside }(76, \\
\text { 13.90) }\end{array}$ & \\
\hline & $\begin{array}{l}\text { Chrysophanol 8-0- } \\
\beta \text {-D-glucoside (77, } \\
14.05)\end{array}$ & \\
\hline & $\begin{array}{l}\text { Physcion } 8-0-\beta-D- \\
\text { glucoside }(\mathbf{8 5} \text {, } \\
\text { 16.12) }\end{array}$ & \\
\hline \multirow[t]{2}{*}{ Rh-03 } & $\begin{array}{l}\text { Sennoside B (34, } \\
6.82)\end{array}$ & \multirow[t]{2}{*}{47 (9.57), 50 (9.96), 53 (10.38), 63 (11.41), 73 (13.52) } \\
\hline & $\begin{array}{l}\text { Sennoside A (46, } \\
9.56)\end{array}$ & \\
\hline
\end{tabular}

A Rh-01 10 represent free anthraquinones, combined anthraquinones, anthranone and its dimers, flavanol and its polymers, gallic acid and gallotannins, stilbene glycosides, naphthalene glycosides, butyrylbenzene and its glycosides, chromones, and flavonoid (flavonol) glycosides in turn. 


\begin{tabular}{|c|c|c|}
\hline Category山 & $\begin{array}{l}\text { Confirmed } \\
\text { component (No., } \\
\text { RT) }\end{array}$ & Remaining component attribution (RT) \\
\hline \multirow[t]{4}{*}{ Rh-04 } & $\begin{array}{l}\text { Cianidanol (11, } \\
\text { 3.24) }\end{array}$ & \multirow[t]{4}{*}{$\begin{array}{l}7(2.75), 8(2.83), 12(3.29), 19(4.25), 20(4.45), 25(5.13), 29 \\
(6.19), 37(7.11), \mathbf{5 4}(10.48), 61(11.29), 69(12.79), 84(16.06)\end{array}$} \\
\hline & $\begin{array}{l}(-) \text {-Epicatechin (18, } \\
4.24)\end{array}$ & \\
\hline & $\begin{array}{l}\text { (-)-Epicatechin } \\
\text { gallate }(\mathbf{3 1}, 6.60)\end{array}$ & \\
\hline & $\begin{array}{l}\text { Procyanidin B2 (14, } \\
3.76)\end{array}$ & \\
\hline Rh-05 & Gallic acid $(4,1.38)$ & $\begin{array}{l}\mathbf{3}(1.19), \mathbf{5}(1.84), \mathbf{6}(2.12), \mathbf{9}(3.00), \mathbf{1 3}(3.40), \mathbf{2 3}(4.76), \mathbf{2 6} \\
(5.18), \mathbf{4 4}(8.83), \mathbf{6 2}(11.34), \mathbf{7 0}(13.09), \mathbf{7 9}(14.66), \mathbf{9 1}(17.74)\end{array}$ \\
\hline Rh-06 & $\begin{array}{l}\text { Resveratroloside } \\
(24,4.86)\end{array}$ & 40 (7.79), 42 (8.01), 43 (8.28), 57 (10.82), 104 (21.72) \\
\hline Rh-07 & $\begin{array}{l}\text { Torachrysone } 8-0- \\
\text { glucoside }(74, \\
13.55)\end{array}$ & $21(4.52), 81(15.44)$ \\
\hline \multirow[t]{3}{*}{ Rh-08 } & $\begin{array}{l}\text { Raspberryketone } \\
\text { glucoside }(\mathbf{1 7}, 4.10)\end{array}$ & \multirow[t]{3}{*}{39 (7.68), 60 (11.16), 75 (13.73), 82 (15.60), 87 (16.61) } \\
\hline & Lindleyin $(33,6.70)$ & \\
\hline & $\begin{array}{l}\text { 4-(4- } \\
\text { Hydroxyphenyl)-2- } \\
\text { butanone }(\mathbf{3 8}, 7.41)\end{array}$ & \\
\hline Rh-09 & $\begin{array}{l}\text { 5-Acetyl-7-hydroxy- } \\
\text { 2-methyl-chromone } \\
(59,11.06)\end{array}$ & 15 (3.82), 16 (3.90), 41 (7.99), 48 (9.68), 56 (10.66), 98 (19.09) \\
\hline Rh-10 & - & 65 (11.72), 67 (12.10), 101 (21.08), 103 (21.31) \\
\hline Others & - & $\begin{array}{l}1(0.81), 2(0.94), 27(5.35), 35(6.96), 45(9.31), 51(10.12), 66 \\
(11.90), 71(13.25), 88(17.26), 94(18.15), 106(22.28), 107 \\
(23.23), 108(23.77)\end{array}$ \\
\hline
\end{tabular}

\section{Evaluation of Five Integration Effects Based on Factor Analysis}

TCM syndromes cannot be completely cured by a single effect frequently, so we explore two or even more effects in an animal model to improve the research efficiency and fitness with actual symptoms. Mice's performance of the first constipation model, selection of E1 and E2 indexes and their pharmacodynamic 
changes were basically consistent with our previous discussion [26]. First black stool time, the number of black stools, fecal weights within $12 \mathrm{~h}$, colonic content weights, organ coefficients of colon and stomach, MTL, SS, VIP and AchE could reflect E1 (Figure S2). Expression levels of TG, $\mathrm{Na}^{+}-\mathrm{K}^{+}-\mathrm{ATP}^{-}$ase, TNF- $\alpha$, IL-1 $\beta$ and grayscale ratios of the aforementioned 9 colonic inflammatory proteins could represent E2 (Figure S3).

The treatment of the second blood stasis syndrome induced by noxious heat also included two aspects of both cooling and activating blood. Model mice appeared obvious symptoms of blood stasis with enlarged spleens and decrescent thymuses $(P<0.001)$, while drug groups' spleens recovered to some extent. On the one hand, we took anal temperatures, HSP-70, SOD, NO, TNF-a, IL-1 $\beta$, IL-6 and the same 9 levels of colonic inflammatory proteins as E3 indicators (Figure S4), which resembled E2 that played an anti-inflammatory role from the perspective of Western medicine. Mice maintained opposite hypothermia $(P<0.001)$ in febrile response to LPS, and some drug groups were able to significantly antagonize the toxic reaction. Compared with the model, most groups down-regulated the contents of inflammatory factors $(P<0.05)$, especially phosphorylated proteins and TLR4 $(P<0.001)$ in high-proportioned ethanolextracted rhubarb groups. LPS could act through the activation of NF-KB signaling pathway mediated by TLR family in connection with pro-inflammatory cytokines [30]. On the other hand, indicators of E4 (Figure S5) covered TT, PT, APTT, FIB, TXB 2 , 6-keto-PGF ${ }_{1 a}$, ratios of $\mathrm{TXB}_{2}$ to 6-keto-PGF ${ }_{1 a}, \mathrm{PGE}_{2}$, ET-1, $\mathrm{Mg}^{2+}$ and $\mathrm{Ca}^{2+}$. The positive group had a better improvement on coagulation four indices, but most of rhubarb groups only showed significant callback to PT and FIB $(P<0.05)$. Ethanol-extracted rhubarb corrected the imbalance of elevatory $\mathrm{TXB}_{2} / 6$-keto-PGF $1 \mathrm{a}(P<0.001)$, which facilitated blood circulation [29]. Prostaglandins could dilate blood vessels whereas endothelins constricted them, which all displayed callback tendency of varying degrees in drug groups.

With regard to the third cholestasis model, as shown in Figure S6, model mice performed visibly declining weights $(P<0.01)$, darkening serum color, intumescent gallbladders and inky bile accompanied by obvious liver damage of which there were dense bleeding points on the surface. The overall state of the positive group presented relatively well recovery, and these lesions also improved to a certain degree in part of rhubarb-treated groups. Whereupon we investigated measurement indexes associated with jaundice including organ coefficients of liver and gallbladder, T-SOD, MDA, GSH, tissue $\mathrm{Fe}^{3+}$, ALT, AST, ALP, serum $\mathrm{Fe}^{3+}, \mathrm{GST}, \mathrm{GGT}, \mathrm{TBIL}, \mathrm{DBIL}$ and TBA to evaluate E5 (Figure S7). The level regulation of oxidative stress in liver tissues $(P<0.05)$ by rhubarb decocted for a long time might be one of the main mechanisms of removing jaundice. Serum liver function levels were generally elevated in model mice and decreased prominently $(P<0.05)$ in most groups after administration except GGT and DBIL.

Factor analysis was carried out for the above pharmacodynamic indexes of five effects to obtain their integration effect values respectively. Referring to the approach we had described [26], comprehensive weight scores of each group were calculated from the scoring coefficient matrix of indicator variables (Tables S6-10), and then Kolmogorov-Smirnov normal distribution test was utilized to examine the results (Tables S11-15). At last, as illustrated in Table 2, there existed significant differences between the model 
and control groups. The therapeutic efficacy of positive drugs emerged very obviously in all five effects while the effectiveness of rhubarb with different preparation was not exactly the same. E1 was conspicuous in groups of $10 \%$ EW-S 35\% EW-L and $80 \%$ EW-L ethanol-L. High-concentration ethanol groups possessed better E2 on the whole. E3 of rhubarb extracted by over $20 \%$ EW was all evident, particularly for a long time, which conformed to the TCM theory that steaming with wine could be good at clearing blood-aspect heat toxin. In contrast, E4 of rhubarb was relatively little and only worked when it was prepared with water or more than $80 \%$ ethanol. 10\% EW-L ethanol-L groups showed prominent E5 among which three groups of 35\% EW-L, 50\% EW-L and 65\% EW-L were the best, suggesting that "wine and water co-decoction" might be more conducive to exert the curative effect. 
Table 2

Integration effects of rhubarb on the basis of factor analysis $(n=6 \sim 8$, mean \pm SD).

\begin{tabular}{|c|c|c|c|c|c|}
\hline Groups & E1 & E2 & E3 & E4 & E5 \\
\hline Control & $(2.08 \pm 0.28)$ & $(-2.53 \pm 1.96)$ & $(-1.24 \pm 3.53)$ & $(1.89 \pm 0.60)$ & $(-1.07 \pm 2.78)$ \\
\hline Model & $\begin{array}{l}(-5.48 \pm \\
1.96)^{\# \# \#}\end{array}$ & $\begin{array}{l}(5.39 \pm \\
3.77)^{\# \# \#}\end{array}$ & $(4.40 \pm 3.62)^{\#}$ & $\begin{array}{l}(-0.89 \pm \\
1.86)^{\# \#}\end{array}$ & $\begin{array}{l}(5.40 \pm \\
2.36)^{\# \# \#}\end{array}$ \\
\hline Positive & $\begin{array}{l}(1.65 \pm \\
3.91)^{\star \star \star}\end{array}$ & $\begin{array}{l}(0.01 \pm \\
2.12)^{\star \star \star}\end{array}$ & $\begin{array}{l}(-0.88 \pm \pm \\
2.32)^{\star \star}\end{array}$ & $(1.70 \pm 1.78)^{*}$ & 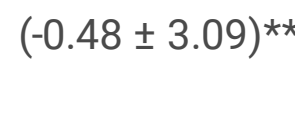 \\
\hline water-S & $\begin{array}{l}(-0.58 \pm \\
2.56)^{\star \star \star}\end{array}$ & $(-0.32 \pm 3.04)^{\star \star}$ & $(2.65 \pm 3.50)$ & $(0.62 \pm 1.77)$ & $(3.60 \pm 1.53)$ \\
\hline water-L & $\begin{array}{l}(-0.35 \pm \\
1.67)^{\star \star \star}\end{array}$ & $(0.12 \pm 0.42)^{\star \star}$ & $(1.22 \pm 2.90)$ & $(1.92 \pm 2.34)^{*}$ & $(2.70 \pm 3.69)$ \\
\hline $10 \%-S$ & $\begin{array}{l}(0.87 \pm \\
0.80)^{\star \star \star}\end{array}$ & $\begin{array}{l}(-0.83 \pm \\
1.89)^{\star \star \star}\end{array}$ & $(2.27 \pm 2.72)$ & $(1.60 \pm 3.66)$ & $(3.22 \pm 1.77)$ \\
\hline $10 \%-L$ & $\begin{array}{l}(0.02 \pm \\
2.53)^{\star \star \star}\end{array}$ & $(0.30 \pm 0.38)^{\star \star}$ & $(0.91 \pm 2.04)$ & $(0.77 \pm 0.63)$ & $(1.10 \pm 4.00)^{\star}$ \\
\hline $20 \%-S$ & $\begin{array}{l}(0.29 \pm \\
2.42)^{\star \star \star}\end{array}$ & $(0.64 \pm 3.21)^{\star \star}$ & $(1.65 \pm 3.36)$ & $(0.08 \pm 1.24)$ & $(1.27 \pm 3.26) \star$ \\
\hline $20 \%-L$ & $\begin{array}{l}(0.52 \pm \\
2.09)^{\star \star \star}\end{array}$ & $(1.93 \pm 2.98) *$ & $(-0.82 \pm 3.59)$ * & $(0.01 \pm 1.80)$ & $(0.45 \pm 2.58)^{\star \star}$ \\
\hline $35 \%-S$ & $\begin{array}{l}(0.09 \pm \\
3.00)^{\star \star \star}\end{array}$ & $(1.22 \pm 3.76)^{\star}$ & $(-0.60 \pm 4.00)^{\star}$ & $(-0.98 \pm 2.97)$ & $(0.35 \pm 3.84)^{\star \star}$ \\
\hline $35 \%-L$ & $\begin{array}{l}(0.80 \pm \\
2.55)^{\star \star \star}\end{array}$ & $(1.48 \pm 3.52)^{\star}$ & $(-0.50 \pm 1.44)^{*}$ & $(-0.33 \pm 0.74)$ & $\begin{array}{l}(-0.97 \pm \\
1.73) \star \star \star\end{array}$ \\
\hline $50 \%-S$ & $\begin{array}{l}(-0.55 \pm \\
2.25)^{\star \star \star}\end{array}$ & $(-0.11 \pm 2.62)^{\star \star}$ & $(-0.27 \pm 3.83)^{\star}$ & $(-0.94 \pm 3.69)$ & $(-0.21 \pm 3.89)^{\star \star}$ \\
\hline $50 \%-\mathrm{L}$ & $\begin{array}{l}(0.13 \pm \\
2.98)^{\star \star \star}\end{array}$ & $(1.10 \pm 2.48)^{*}$ & $(-0.82 \pm 1.42)^{*}$ & $(-0.17 \pm 2.32)$ & $\begin{array}{l}(-1.01 \pm \\
2.29)^{\star \star \star}\end{array}$ \\
\hline $65 \%-S$ & $(-1.84 \pm 2.63)^{\star \star}$ & $\begin{array}{l}(-1.10 \pm \\
3.09)^{\star \star \star}\end{array}$ & $(-0.61 \pm 2.24)^{\star}$ & $(-0.69 \pm 3.38)$ & $\begin{array}{l}(-0.32 \pm \\
2.39)^{\star \star \star}\end{array}$ \\
\hline $65 \%-L$ & $(-0.29 \pm 3.30) \star \star$ & $\begin{array}{l}(-0.53 \pm \\
2.24)^{\star \star \star}\end{array}$ & $\begin{array}{l}(-1.92 \pm \\
2.01)^{\star \star}\end{array}$ & $(-0.66 \pm 2.06)$ & $\begin{array}{l}(-1.55 \pm \\
3.29)^{\star \star \star}\end{array}$ \\
\hline $80 \%-S$ & $(-0.67 \pm 3.18)^{\star \star}$ & $\begin{array}{l}(-1.38 \pm \\
2.57)^{\star \star \star}\end{array}$ & $(-0.12 \pm 3.36)^{*}$ & $(-0.52 \pm 2.21)$ & $(0.14 \pm 3.88)^{\star \star}$ \\
\hline $80 \%-\mathrm{L}$ & $\begin{array}{l}(0.47 \pm \\
3.49)^{\star \star \star}\end{array}$ & $(-0.76 \pm 3.02) \star \star$ & $\begin{array}{l}(-1.71 \pm \\
2.02)^{\star \star}\end{array}$ & $(0.89 \pm 2.31)$ & $(-0.37 \pm 3.48)^{\star \star}$ \\
\hline
\end{tabular}




\begin{tabular}{|c|c|c|c|c|c|}
\hline Groups & E1 & E2 & E3 & E4 & E5 \\
\hline $90 \%-S$ & $\begin{array}{l}(1.00 \pm \\
3.00)^{\star \star \star}\end{array}$ & $\begin{array}{l}(-1.43 \pm \\
2.71)^{\star \star \star}\end{array}$ & $\begin{array}{l}(-2.25 \pm \\
2.69)^{\star \star}\end{array}$ & $(1.58 \pm 1.49)^{\star}$ & $(-0.76 \pm 3.76)^{\star \star}$ \\
\hline $90 \%-\mathrm{L}$ & $\begin{array}{l}(0.47 \pm \\
2.38)^{\star \star \star}\end{array}$ & $\begin{array}{l}(-2.11 \pm \\
1.25)^{\star \star \star}\end{array}$ & $\begin{array}{l}(-2.40 \pm \\
3.29)^{\star \star}\end{array}$ & $(0.66 \pm 1.79)$ & $\begin{array}{l}(-0.42 \pm \\
3.00)^{\star \star \star}\end{array}$ \\
\hline $\begin{array}{l}\text { ethanol- } \\
\text { S }\end{array}$ & $\begin{array}{l}(1.42 \pm \\
3.08)^{\star \star \star}\end{array}$ & $\begin{array}{l}(-3.39 \pm \\
1.99)^{\star \star \star}\end{array}$ & $\begin{array}{l}(-1.96 \pm \\
2.34)^{\star \star}\end{array}$ & $(1.22 \pm 2.91)$ & $(-0.49 \pm 3.86)^{\star \star}$ \\
\hline ethanol- & $\begin{array}{l}(2.19 \pm \\
3.40)^{\star \star \star}\end{array}$ & $\begin{array}{l}(-2.61 \pm \\
0.61)^{\star \star \star}\end{array}$ & $\begin{array}{l}(-1.25 \pm \\
1.54)^{\star \star}\end{array}$ & $(0.98 \pm 1.44)$ & $(0.08 \pm 3.96)^{\star \star}$ \\
\hline \multicolumn{6}{|c|}{${ }^{\#} P<0.05$, \#\# $P<0.01$, \#\# $P<0.001$ compared with the Control; } \\
\hline \multicolumn{6}{|c|}{ * $P<0.05$, ** $P<0.01$, $\star \star \star ~ P<0.001$ compared with the Model. } \\
\hline
\end{tabular}

The advanced BP-ANN mimics neurons based on the structure and function of the biological brain with high prediction accuracy and optimization ability [31,32]. It can be intelligently applied in the fitting correlation analysis to help us determine the contribution degree of each chemical component to the corresponding efficacy. So, we tried to objectively associate as many components detected with efficacies as possible. The reasonable quantity-effect correlation method was established by repeatedly debugging parameters as follows.

(1) The number of hidden layers: Theoretically, the more hidden layers, the stronger the ability to fit functions is. However, in fact, many layers are apt to cause overfitting as well as increase training difficulty of convergence. On the premise of setting 108 nodes in the input layer and outputting 1 node, we explored the fitting consequence at 1,2 and 3 layers. The number of nodes in different hidden layers was randomly set to 10 , and relative errors between the predicted and true values were consequently compared by means of repeat for 20 runs. It could be seen in Figure 3 that, although the relative error values of double hidden layers were not optimal, they were more stable and not easy to appear local extremum. Three hidden layers had maximum negative values combined with observing the predicted values of test samples (Table S16). So, we adopted 2 hidden layers for the next step.

(2) The number of neurons per hidden layer: According to empirical formulas and the setting principle of hidden neurons, in the range of 108 input nodes to 1 output node, the number of neurons was set every 20 values (i.e., 20, 40, 60, 80, 100), and then added 5, 10 and 15 from 0 to 20,70 and 75 from 60 to 80 , 130, 160 and 190 behind 100 . The second hidden layer kept pace with the first one. Five sets of data from three test samples were acquired by 5 runs under each number of neurons (Table S17). Synthetically in 
view of the predicted, true and their relative error values, we set the number of neurons at 20 due to its more steady relative errors on the whole.

(3) Learning rate: In general, it tends to choose small adaptive learning rates to ensure the stability of the system, but too small learning rates will lead to a very long training time. In order to obtain better results, we ran test samples for five times severally when the learning rate was $0.01,0.02,0.03,0.04,0.05,0.06$, $0.07,0.08,0.09,0.1,0.2,0.3,0.4,0.5,0.6,0.7$ and 0.8 (Table S18). It was found that there were no significant trend changes. Relatively speaking, the learning rate of 0.02 could make BP algorithm with gradient descent preferable.

(4) Running effect: We adjusted the display frequency to 100 , training times to 1000 and the minimum error of training target to 0.0000001 for further accurate prediction of this method. After repeated debugging, the final running program was demonstrated in Figure S8 (a). All training samples and corresponding integration effect values were substituted into respective optimized neural networks. The training would stop when the mean squared error was less than its set point or the gradient reached its set value, and it was forced to end as well when errors did not decrease but rise continuously for 6 times by validation checks of the generalization capability. As a result, the fitting correlation effects were all excellent (Figure 4).

(5) Decision weight calculation: The above neural network training results only reflected the relationship among neurons. What's more, we needed to clarify the relative importance of each input factor to output information. Hence, the following neural network learning algorithms were sequentially used to work out decision weights of input components.

(The input layer: $n=108$, the first hidden layer: $m=20$, the second hidden layer: $p=20$, the output layer: I $=1$.)

Impact of the $o$ unit in the second hidden layer on the $k$ output relative to all units:

$F_{o k}=\left|w_{o k}\right| / ? ?\left|w_{o k}\right| o=1,2 \ldots p, k=1,2 \ldots l$

Impact of the $j$ unit in the first hidden layer on the $o$ unit in the second hidden layer relative to all units:

$F_{j o}=\left|w_{j o}\right| / ? ?\left|w_{j o}\right| j=1,2 \ldots m, o=1,2 \ldots p$

Impact of the $i$ unit in the input layer on the $j$ unit in the first hidden layer relative to all units:

$F_{i j}=\left|w_{i j}\right| / ? ?\left|w_{i j}\right| i=1,2 \ldots n, j=1,2 \ldots m$

Thus, impact of the $i$ input on the $k$ output: 


$$
F_{i k}=F_{i j} * F_{j o} * F_{o k}
$$

Decision weights of input components could be expressed as:

$$
S_{i}=F_{i k} / ? F_{i k}
$$

\section{The Main Bioactive Components for Five Effects on the Basis of Association Analysis of Components} and Effects

We analyzed the components whose contribution was greater than 0.01 ranking from the ultimate decision weight Table S19, which were 30 in E1 (Nos. $42>\mathbf{2 1}>\mathbf{3 5}>\mathbf{3 7}>\mathbf{3 4}>\mathbf{4 6}>\mathbf{1 1}>\mathbf{9 8}>\mathbf{3 6}>\mathbf{2 7}>\mathbf{5 9}$ $>88>89>18>108>78>105>72>6>55>106>45>61>51>90>32>41>95>38>107$ ), 28 in E2 (Nos. $42>7>107>83>29>28>61>98>108>8>80>35>58>81>40>15>92>33>106>36>$ $50>79>11>100>57>19>101>39$ ), 35 in E3 (Nos. $24>18>12>86>72>37>51>80>26>55>$ $34>21>16>46>97>42>22>33>88>28>92>27>2>67>70>59>36,44>90>1>82>6>73>$ 4, 30), 32 in E4 (Nos. $40>81>56>75>69>53>9>64>100>58>49>29>74>83>90>52>63>$ $42>66>106>67>48>31>62>71>89>87>92>20>60>46>36$ ) and 33 in E5 (Nos. $19>55>62$ $>12>33>52>22>35>87>64>69>95>59>42>88>46>43>25>107>72>29>104>71>94>$ $89>99>92>81>36>45>58>108>102$ ). Table 3 summarized the total contribution of each category of rhubarb components. Thereinto, the top 10 of total weights in five effects and of each effect should be focused on respectively (Table S20-25).

Table 3. Total contribution of each category of rhubarb components with weights over 0.01 .

\begin{tabular}{ccccccc}
\hline Category $^{\mathbf{4}}$ & E1 & E2 & E3 & E4 & E5 & Total \\
\hline Rh-02 & $0.05621^{(1)}$ & $0.06679^{(2)}$ & $0.11795^{(1)}$ & $0.09344^{(1)}$ & $0.10226^{(1)}$ & 0.43665 \\
Rh-04 & $0.05001^{(2)}$ & $0.06684^{(1)}$ & $0.04032^{(3)}$ & $0.04748^{(2)}$ & $0.06193^{(2)}$ & 0.26658 \\
Rh-06 & $0.01966^{(6)}$ & $0.03350^{(3)}$ & $0.02689^{(5)}$ & $0.02719^{(5)}$ & $0.03299^{(3)}$ & 0.14023 \\
Rh-03 & $0.02866^{(5)}$ & $0.01069^{(7)}$ & $0.03469^{(4)}$ & $0.03579^{(3)}$ & $0.01110^{(8)}$ & 0.12093 \\
Rh-01 & $0.04255^{(3)}$ & $0.01052^{(9)}$ & $0.01023^{(10)}$ & $0.02507^{(8)}$ & $0.03212^{(4)}$ & 0.12049 \\
Rh-09 & $0.03611^{(4)}$ & $0.02220^{(4)}$ & $0.02266^{(6)}$ & $0.02550^{(7)}$ & $0.01157^{(7)}$ & 0.11804 \\
Rh-05 & $0.01059^{(8)}$ & $0.01060^{(8)}$ & $0.05335^{(2)}$ & $0.02418^{(9)}$ & $0.01423^{(6)}$ & 0.11295 \\
Rh-08 & $0.01014^{(9)}$ & $0.02084^{(5)}$ & $0.02138^{(7)}$ & $0.03495^{(4)}$ & $0.02452^{(5)}$ & 0.11183 \\
Rh-07 & $0.01925^{(7)}$ & $0.01101^{(6)}$ & $0.01243^{(8)}$ & $0.02694^{(6)}$ & $0.01023^{(9)}$ & 0.07986 \\
Rh-10 & $0.00000^{(10)}$ & $0.01005^{(10)}$ & $0.01060^{(9)}$ & $0.01113^{(10)}$ & $0.00000^{(10)}$ & 0.03178 \\
\hline
\end{tabular}

A $\mathrm{Rh}$-01 10 represent free anthraquinones, combined anthraquinones, anthranone and its dimers, flavanol and its polymers, gallic acid and gallotannins, stilbene glycosides, naphthalene glycosides, butyrylbenzene and its glycosides, chromones, and flavonoid (flavonol) glycosides in tum.

The ordinal number after the values represents the order in each efficacy.

Blue highlights the universality character of different efficacies of rhubarb; yellow highlights the individuality character of different efficacies of rhubarb.

The hypothesis of "additive effect" of medicinal herbs was put forward in 2014 [33, 34]. That is, the assemblage or superposition of "effective forms" is the core chemical essence of medicinal herbs. 
Compounds with identical mother nuclei structure are grouped together and may have the same pharmacological target. Both the "additive effect" of various components on single target and the "synergy effect" on multi-targets, even universality and individuality characters of different efficacies, can be well reflected in the Table 3 above, which is also the essence of fuzzy identification our team proposed for new interpretations to pharmacodynamic components and action mechanisms of a medicinal herb.

In this study, we quantified the "additive effect" of these chemical groups. Through specific values, we can see that combined anthraquinones, flavanol and its polymers might be the universality character to the multi-functional properties of rhubarb, reflecting the correlation among multiple efficacies to some extent. For example, chrysophanol glucosides rank the 18th, 4th, 4th, 8th and 10th severally in E1 5. Their aglycones, namely free anthraquinones, possess strong bacteriostasis and notably affect the activation of lipid inflammatory mediators [35]. There are currently many evidences that rhubarb anthraquinones can treat constipation, ischemic lesions [36], sepsis [37] and other inflammation. Especially now in the suppression of hepatobiliary diseases [38, 39], Kehuanglidan Capsule has completed the treatment of chronic viral hepatitis jaundice in the drug clinical trial registration and information public platform (www.chinadrugtrials.org.cn), and its dominant ingredients are the rhubarb anthraquinones. Some literature speculated that these anthraquinone glycosides might revert to free type ones through metabolic transformation in vivo to exert various pharmacological actions. In addition, flavanols such as Cianidanol and (-)-Epicatechin rank in the top 10 of E1 5. Catechins contribute to improve metabolic changes caused by a high-fat diet [40], and can maintain normal blood circulation by reducing the adhesion of platelets [41] and efficaciously shorten icteric period by lowering serum bilirubin levels [42]. Procyanidins are the polymers of flavanols. They also have great contribution for E1, E2, E3 and $\mathrm{E} 5$, showing protective effects on the digestive system for the treatment of gastrointestinal diseases, cholecystitis, constipation or diarrhea, etc. In particular, oligomeric procyanidins are the most in the top 10 of E2, which are the internationally recognized natural antioxidant to scavenge free radicals.

With regard to individuality characters of different efficacies, stilbene glycosides are important to E2 5 that are closely linked to anti-bacteria, antiphlogosis and other aspects [43]. Stilbene compounds are always a momentous part of rhubarb chiefly for anti-hyperlipidemia and antioxidation [38]. Resveratrol glucosides are the representative ranking high in these efficacies, which can alleviate LPS stimulation by inhibiting TNF-a and macrophages from producing NO [44]. Sennosides belong to anthranone dimers not only for defaecation (E1), but also for detoxifying to treat bacillary dysentery, epidemic hemorrhagic fever (E3) and postpartum milk return in clinic (E4). Free anthraquinones, mainly Emodin, Physcion, Rhein or their derivates, act directly in E1 and E5. Chromones appear in the top 10 of E1 and E2, which have been proven to remove cholesterol, inflammation and so on [38]. A kind of gallotannins ranks the 9th in E3. Gallotannins are generally characterized by potent antivirus, astringency and hemostasis [38]. E3 of rhubarb is related to clinical blood loss syndrome, and grey relational analysis has attested to a big impact of tannins and anthraquinones on the hemostatic function [45]. Butyrylbenzene and its glycosides may play a certain protective role on the cardiovascular system (E4). 
To sum up, from the perspective of both components whose contribution $>0.01$ and the top 10 components, we revealed the universality and individuality characters of five effects. As intuitively shown in Figure 5, combined anthraquinones, flavanol and its polymers might be the universality character to the multi-functional properties of rhubarb while their individuality characters were listed severally. There are some inadequacies of lacking experimental validation on effective constituents, which would be designed in our subsequent work. And if these active components act alone or exist in other drugs, whether they can also contribute to the same efficacy still needs to be further studied.

\section{Conclusion}

This study proposed a generally applicable strategy to fuzzily identify bioactive components for different efficacies of rhubarb by BP-ANN association analysis of UPLC-Q-TOF/MS ${ }^{\mathrm{E}}$ and integrated effects. Based on fuzzy chemical identification, we attributed more than 100 components into ten categories and then established three mouse models to explore the five efficacies recorded in Chinese Pharmacopoeia. It suggested that combined anthraquinones, flavanol and its polymers might be the universality character to the multi-functional properties of rhubarb while stilbene glycosides, anthranone and its dimers, free anthraquinones, chromones, gallic acid and gallotannins, butyrylbenzene and its glycosides contributed to rhubarb's individuality characteristics. These results will provide scientific evidence to support the expansion of herbal use in clinical applications as well as the further development of some products of rhubarb. And this approach should also be useful for investigating the bioactive constituents of other medicinal herbs or natural functional products.

\section{Abbreviations}

AchE, acetyl cholinesterase; Adr, adrenalin hydrochloride; ALP, alkaline phosphatase; ALT, glutamic-pyruvic transaminase; ANIT, a-naphthyl isothiocyanate; APTT, activated partial thromboplastin time; AST, glutamic-oxalacetic transaminase; BP-ANN, back propagation artificial neural network; $\mathrm{Ca}^{2+}$, calcium ion; CAS, chemical abstracts service; DBIL, direct bilirubin; DMSO, dimethyl sulfoxide; E1, removing accumulation with purgation; E2, clearing heat and purging fire; E3, cooling blood and detoxifying; $E 4$, eliminating blood stasis to remove obstruction in channels; $E 5$, disinhibiting dampness and removing jaundice; ELISA, enzyme-linked immunosorbent assay; ET-1, endothelin-1; EW, ethanolwater; $\mathrm{Fe}^{3+}$, ferric ion; FIB, fibrinogen; GAPDH, glyceraldehyde-3-phosphate dehydrogenase; GGT, $\mathrm{Y}^{-}$ glutamyl transpeptidase; GSH, glutathione; GST, glutathione sulfhydryl transferase; HSP-70, heat shock protein-70; IL, interleukin; L, long time; LPS, lipopolysaccharide; MDA, malondialdehyde; $\mathrm{Mg}^{2+}$, magnesium ion; MTL, motilin; $\mathrm{Na}^{+}-\mathrm{K}^{+}$-ATPase, sodium potassium pump; NO, nitric oxide; NS, normal saline; PCA, principal component analysis; (p-)ERK, (phosphorylated-)extracellular regulated protein kinase; PG, prostaglandin; ( $p$-)JNK, (phosphorylated-)c-Jun N-terminal kinase; (p-)NF-kB p65, (phosphorylated-)nuclear factor-kappaB protein65; (p-)p38, (phosphorylated-)protein38; PT, prothrombin 
time; QC, quality control; RT, retention time; S, Short time; SD, standard deviation; SS, somatostatin; TBA, total bile acid; TBIL, total bilirubin; TCM, traditional Chinese medicine; TG, triglyceride; TLR4, toll-like receptor4; TNF, tumor necrosis factor; (T-)SOD, (total-)superoxide dismutase; TT, thrombin time; $\mathrm{TXB}_{2}$, thromboxane $\mathrm{B}_{2}$; UPLC-Q-TOF/MS ${ }^{\mathrm{E}}$, ultra-performance liquid chromatography/quadrupole time-of-flight mass spectrometry for every data; UV, ultraviolet; VIP, vasoactive intestinal peptide.

\section{Declarations}

\section{Supplementary Information}

The online version contains supplementary material available at https://doi.org/.

\section{Authors' contributions}

Jia-Qian Chen and Yu-Ping Tang conceived of and proposed the idea, reviewed literature. Jia-Qian Chen, Yan-Yan Chen, Hui-Juan Tao, Zong-Jin Pu and Xu-Qin Shi carried out the experiments, participated in the study design and wrote the original draft. Xia Du and Er-Xin Shang performed back propagation artificial neural network association analysis. Xia Du, Shi-Jun Yue, Gui-Sheng Zhou, Er-Xin Shang, Yu-Ping Tang and Jin-Ao Duan contributed to revising the article.

\section{Funding}

This work was financially supported by National Key R\&D Program of China (2019YFC1711000), the National Natural Science Foundation of China (81974525), and Youth Talent Project Funded by Shaanxi Higher Education Association for Science and Technology (20180307). This research was also financially supported by Subject Innovation Team of Shaanxi University of Chinese Medicine (2019-YL10) and the Youth Innovation Team of Shaanxi Universities (2020).

\section{Availability of data and materials}

The research data generated from this study are included in the article and additional files.

\section{Ethics approval and consent to participate}

Experimental animal protocols were approved by the Institutional Animal Care and Use Committee of Nanjing University of Chinese Medicine and the procedures were conformed to the Guide for the Care and Use of Laboratory Animals.

\section{Consent for publication}

All authors critically reviewed the content of the manuscript. The consent for publication was obtained from all authors. 


\section{Competing interests}

No potential conflict of interest was reported by the authors. The authors declare no conflict of interest.

\section{References}

1. Agatonovic-Kustrin S, Doyle E, Gegechkori V, Morton DW. High-performance thin-layer chromatography linked with (bio)assays and FTIR-ATR spectroscopy as a method for discovery and quantification of bioactive components in native Australian plants. J Pharm Biomed Anal. 2020;184:113208.

2. Ayoola-Oresanya IO, Sonibare MA, Gueye B, Paliwal R, Abberton MT, Morlock GE. Effect-directed profiling and identification of bioactive metabolites from field, in vitro-grown and acclimatized Musa spp. accessions using high-performance thin-layer chromatography-mass spectrometry. $J$ Chromatogr A. 2020;1616:460774.

3. Cao YJ, Pu ZJ, Tang YP, Shen J, Chen YY, Kang A, Zhou GS, Duan JA. Advances in bio-active constituents, pharmacology and clinical applications of rhubarb. Chin Med. 2017;12:36-47.

4. Chen JQ, Chen YY, Tang YP, Zhou GS, Shang EX, Duan JA. Multifunctional regularity of Rhei Radix et Rhizoma in ancient and modern medicine. Chin Tradit Herb Drugs. 2019;50:1485-92.

5. Chen JQ, Chen YY, Tao HJ, Pu ZJ, Shi XQ, Zhang J, Tan YJ, Yue SJ, Zhou GS, Shang EX, Tang YP, Duan JA. An integrated metabolomics strategy to reveal dose-effect relationship and therapeutic mechanisms of different efficacy of rhubarb in constipation rats. J Pharm Biomed Anal. 2020;177:112837.

6. Chen JQ, Li DW, Chen YY, Tao HJ, Pu ZJ, Zhang J, Tan YJ, Shi XQ, Yue SJ, Zhou GS, Tang YP, Duan JA. Elucidating dosage-effect relationship of different efficacy of rhubarb in constipation model rats by factor analysis. J Ethnopharmacol. 2019;238:111868.

7. Cheng Y, Zhang HQ, Qu LJ, He Y, Routledge MN, Gong YY, Qiao BL. Identification of rhein as the metabolite responsible for toxicity of rhubarb anthraquinones. Food Chem. 2020;331:127363.

8. Choi RJ, Ngoc TM, Bae K, Cho HJ, Kim DD, Chun J, Khan S, Kim YS. Anti-inflammatory properties of anthraquinones and their relationship with the regulation of P-glycoprotein function and expression. Eur J Pharm Sci. 2013;48:272-81.

9. Du T. Overview of the biological activities and application prospects of catechin and its polymers. Biotech World. 2013;3:89-90.

10. Fardet A, Llorach R, Martin JF, Besson C, Lyan B, Pujos-Guillot E, Scalbert A. A liquid chromatographyquadrupole time-of-flight (LC-QTOF)-based metabolomic approach reveals new metabolic effects of catechin in rats fed high-fat diets. J Proteome Res. 2008;7:2388-98.

11. Gao D, Zeng LN, Zhang P, Ma ZJ, Li RS, Zhao YL, Zhang YM, Guo YM, Niu M, Bai ZF, Xiao XH, Gao WW, Wang JB. Rhubarb anthraquinones protect rats against mercuric chloride ( $\mathrm{HgCl} 2)$-induced acute renal failure. Molecules. 2016;21:298-309. 
12. Gong XH, Li Y, Zhang RQ, Xie XF, Peng C, Li YX. The synergism mechanism of Rhubarb Anthraquinones on constipation elucidated by comparative pharmacokinetics of Rhubarb extract between normal and diseased rats. Eur J Drug Metab Pharmacokinet. 2015;40:379-88.

13. Hao HP, Cui N, Wang GJ, Xiang BR, Liang Y, Xu XY, Zhang H, Yang J, Zheng CN, Wu L, Gong P, Wang W. Global detection and identification of nontarget components from herbal preparations by liquid chromatography hybrid ion trap time-of-flight mass spectrometry and a strategy. Anal Chem. 2008;80:8187-94.

14. Jiao DH, Du SJ. Rhubarb Research. Shanghai: Shanghai Scientific \& Technical Publishers; 2000.

15. Lai F, Zhang Y, Xie DP, Mai ST, Weng YN, Du D, Wu GP, Zheng JX, Han Y. A systematic review of rhubarb (a traditional Chinese medicine) used for the treatment of experimental sepsis. Evid Based Complement Alternat Med. 2015;2015:131283.

16. Li C, Xu F, Xie DM, Jing Y, Shang MY, Liu GX, Wang X, Cai SQ. Identification of absorbed constituents in the rabbit plasma and cerebrospinal fluid after intranasal administration of Asari Radix et Rhizoma by HS-SPME-GC-MS and HPLC-APCIIT-TOF-MS ${ }^{n}$. Molecules. 2014;19:4857-79.

17. Li C, Zhang WY, Yu Y, Cheng CS, Han JY, Yao XS, Zhou H. Discovery of the mechanisms and major bioactive compounds responsible for the protective effects of Gualou Xiebai Decoction on coronary heart disease by network pharmacology analysis. Phytomedicine. 2019;56:261-8.

18. Li S, Wang L, Du ZF, Jin SN, Song CW, Jia SL, Zhang Y, Jiang HL. Identification of the lipid-lowering component of triterpenes from Alismatis rhizoma based on the MRM-based characteristic chemical profiles and support vector machine model. Anal Bioanal Chem. 2019;411:3257-68.

19. Li XX, Qin JZ. Modulation of Toll-interleukin 1 receptor mediated signaling. J Mol Med. 2005;83:258-66.

20. Lin YL, Wu CF, Huang YT. Effects of rhubarb on migration of rat hepatic stellate cells. J Gastroenterol Hepatol. 2009;24:453-61.

21. Liu AJ, Song L, Li Y, Zhang XG, Chen ZX, Huang LB, Zhang HF, Zheng GQ. Active compounds of rhubarb root and rhizome in animal model experiments of focal cerebral ischemia. Evid Based Complement Alternat Med. 2015;2015:10546.

22. Liu DM, Yang D, Zhou CY, Wu JS, Zhang GL, Wang P, Wang F, Meng XL. Aloe-emodin induces hepatotoxicity by the inhibition of multidrug resistance protein 2. Phytomedicine. 2020;68:153148.

23. Matsuda H, Kageura T, Morikawa T, Toguchida I, Harima S, Yoshikawa M. Effects of stilbene constituents from rhubarb on nitric oxide production in lipopolysaccharide-activated macrophages. Bioorg Med Chem Lett. 2000;10:323-7.

24. Moon MK, Kang DG, Lee JK, Kim JS, Lee HS. Vasodilatory and anti-inflammatory effects of the aqueous extract of rhubarb via a NO-cGMP pathway. Life Sci. 2006;78:1550-7.

25. Móricz AM, Häbe TT, Ott PG, Morlock GE. Comparison of high-performance thin-layer with overpressured layer chromatography combined with direct bioautography and direct analysis in real time mass spectrometry for tansy root. J Chromatogr A. 2019;1603:355-60. 
26. Qian WQ, Wu WL, Kang Y, Wang YQ, Yang P, Deng YR, Ni CJ, Huang JM. Comprehensive identification of minor components and bioassay-guided isolation of an unusual antioxidant from Azolla imbricata using ultra-high performance liquid chromatography-quadrupole time-of-flight mass spectrometry combined with multicomponent knockout and bioactivity evaluation. J Chromatogr A. 2020;1609:460435.

27. Qu C, Wang LY, Jin WT, Tang YP, Jin Y, Shi XQ, Shang LL, Shang EX, Duan JA. Comparative analysis of the effects of hydroxysafflor yellow $A$ and anhydrosafflor yellow $B$ in safflower series of herb pairs using prep-HPLC and a selective knock-out approach. Molecules. 2016;21:1480-99.

28. Qu C, Wang LY, Lin H, Shang EX, Tang YP, Yue SJ, Jin Y, Tao WW, Li SP, Hua YQ, Liu P, Su SL, Zhou HP, Qian DW, Duan JA. Hierarchical identification of bioactive components in a medicinal herb by preparative high-performance liquid chromatography and selective knock-out strategy. J Pharm Biomed Anal. 2017;135:206-16.

29. Shen J, Mo X, Tang YP, Zhang L, Pang HQ, Qian YF, Chen YY, Tao WW, Guo S, Shang EX, Zhu SQ, Ding YH, Guo JM, Liu P, Su SL, Qian DW, Duan JA. Analysis of herb-herb interaction when decocting together by using ultra-high-performance liquid chromatography-tandem mass spectrometry and fuzzy chemical identification strategy with poly-proportion design. J Chromatogr A. 2013;1297:16878.

30. Su CN, Li CH, Sun K, Li WJ, Liu RX. Quantitative analysis of bioactive components in walnut leaves by UHPLC-Q-Orbitrap HRMS combined with QAMS. Food Chem. 2020;331:127180.

31. Tang YP, Shang EX, Chen YY, Yue SJ, Shi XQ, Dong TX, Tsim WK, Duan JA. Modern research approaches and strategies for compatibility effects and efficacy components of herbal pairs. Acta Pharm Sin. 2019;54:1564-73.

32. Wang J, Wang F, Liu Y, Xu J, Lin H, Jia B, Zuo W, Jiang Y, Hu L, Lin F. Multiple linear regression and artificial neural network to predict blood glucose in overweight patients. Exp Clin Endocrinol Diabetes 2016:124:34-8.

33. Wang LY, Liu TangYP, Zhu X, Tao M, Li WW, Duan WX. JA. Effects of ferulic acid on antioxidant activity in Angelicae Sinensis Radix, Chuanxiong Rhizoma, and their combination. Chin JNat Med. 2015;13:401-8.

34. Wang M, Hu GN, Tian Y, Zhang ZJ, Song R. Influence of wine-processing on the pharmacokinetics of anthraquinone aglycones and glycosides from rhubarb in hyperlipidemic hamsters. RSC Adv. 2016;6:24871-9.

35. Xiang H, Zuo JX, Guo FY, Dong DS. What we already know about rhubarb: a comprehensive review. Chin Med. 2020;15:88-109.

36. Xie J, Xiong J, Ding LS, Chen L, Zhou H, Liu L, Zhang ZF, Hu XM, Luo P, Qing LS. A efficient method to identify cardioprotective components of Astragali Radix using a combination of molecularly imprinted polymers-based knockout extract and activity evaluation. J Chromatogr A. 2018;1576:108. 
37. Xu F, Yang DH, Shang MY, Wang X, Cai S. Effective forms, additive effect, and toxicities scattering effect of pharmacodynamic substances of TCMs some reflections evoked by the study on the metabolic disposition of traditional chinese medicines (TCM). World Sci Technol/Mod Tradit Chin Med Mater Med. 2014;16:688-703.

38. Zhang CE, Niu M, Li RY, Feng WW, Ma X, Dong Q, Ma ZJ, Li GQ, Meng YK, Wang Y, Yin P, He LZ, Li YM, Tan P, Zhao YL, Wang JB, Dong XP, Xiao XH. Untargeted metabolomics reveals dose-response characteristics for effect of rhubarb in a rat model of cholestasis. Front Pharmacol. 2016;7:85-99.

39. Zhang XJ, Zhou JP, Xu Y. Optimized parameters for the preparation of silk fibroin drug-loaded microspheres based on the response surface method and a genetic algorithm-backpropagation neural network model. J Biomed Mater Res B Appl Biomater. 2021;109:6-18.

40. Zhang Y, Xiong B, Yi N, Su HB, Liu H. Advances in research on pharmacological activities of flavanol compounds. J Food Saf Qual. 2015;6:1055-60.

41. Zhao XF, Zheng XH, Fan TP, Li ZJ, Zhang YY, Zheng JB. A novel drug discovery strategy inspired by traditional medicine philosophies. Science. 2015;6219:38-40.

42. Zhou X, Song BA, Jin LH, Hu DY, Diao CL, Xu GF, Zou ZH, Yang S. Isolation and inhibitory activity against ERK Phosphorylation of hydroxyanthraquinones from rhubarb. Bioorg Med Chem Lett. 2006;16:563-8.

43. Zhu H, Liu X, Zhu TT, Wang XL, Qin KM, Pei K, Cai BC. UHPLC-MS/MS method for the simultaneous quantitation of five anthraquinones and gallic acid in rat plasma after oral administration of prepared rhubarb decoction and its application to a pharmacokinetic study in normal and acute blood stasis rats. J Sep Sci. 2017:40:2382-9.

44. Zhu H, Liu X, Zhu TT, Wang XL, Zhu XC, Cai BC. Determination of the related components in prepared rhubarb decoction and its pharmacodynamics study. J Nanjing Univ Tradit Chin Med. 2017;33:4705.

45. Zhu ST, Lei P, Li XZ, Li YL. Grey relational analysis fingerprint and hemostatic function of Rheum palmatum L. Cent S Pharm. 2009;7:55-8.

\section{Figures}


Chemical Component Study

Herb medicine

| Different solvents
Integration Effect Evaluation

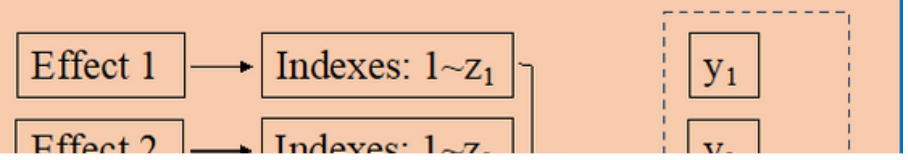

\section{Figure 1}

Summary diagram of the developed strategy and approach.

\section{Figure 2}

Networks of compound groups taking high-content anthraquinones (A), and flavanol and its polymers, gallic acid and gallotannins (B) for examples (Inside the circle is the molecular weight determined by molecular ion peaks in positive and negative ion modes; Inside the box is the serial number of each component of rhubarb samples, and red means components confirmed by chemical references).

\section{Figure 3}

Relative errors between the predicted and true values of $90 \%$ EW-L (A), ethanol-S (B) and ethanol-L (C) groups influenced by 1,2 and 3 hidden layers. 


\section{Figure 4}

Running effect diagrams of the neural network performance $(A)$, training state $(B)$, regression analysis (C), comparison between the predicted and true values ( $D$ : the line is the prediction curve and " $\star$ " denotes the true value of each group) belonging to $\mathrm{E} 1$ as an example (E2 5 are shown in Figure S8 b e).

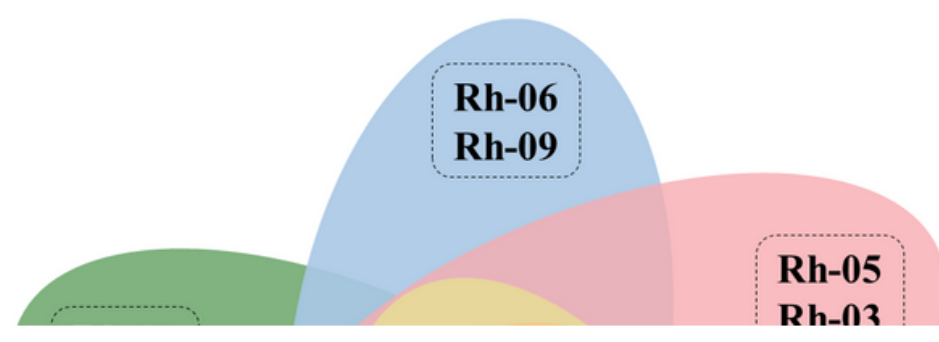

E1: removing accumulation with purgation

E2: clearing heat and purging fire

E3: cooling blood and detoxifying

E4: eliminating blood stasis to remove obstruction in channels

\section{Figure 5}

Venn diagram of the universality and individuality characters for the different efficacies of rhubarb. (Rh01, free anthraquinones; Rh-02, combined anthraquinones; Rh-03, anthranone and its dimers; Rh-04, flavanol and its polymers; Rh-05, gallic acid and gallotannins; Rh-06, stilbene glycosides; Rh-08, butyrylbenzene and its glycosides; Rh-09, chromones.)

\section{Supplementary Files}

This is a list of supplementary files associated with this preprint. Click to download.

- SupplementaryMaterials.docx 